\title{
Oligochaeta (Annelida) de ambientes aquáticos continentais do Estado do Mato Grosso do Sul (Brasil)
}

\author{
Alice Michiyo Takeda', Daniele Sayuri Fujita², Flávio Henrique Ragonha3 \\ Danielle Katharine Petsch ${ }^{3} \&$ Maria Célia Montanholi-Martins ${ }^{4}$
}

\begin{abstract}
1. Universidade Estadual de Maringá. DBI/NUPELIA/PEA, Av. Colombo, 5790, 87020-900 Maringá, PR, Brasil. (alicemtakeda@yahoo.com.br)
2. Instituto Federal de Educação, Ciência e Tecnologia de Roraima, Campus Amajari, Vicinal de acesso que liga a balsa de Aparecida à Vila Brasil, Km $03,69343-000$ Amajari, RR, Brasil.

3. Programa de Pós-Graduação em Ecologia de Ambientes Aquáticos Continentais - Universidade Estadual de Maringá, Av. Colombo, 5790, 87020-900 Maringá, PR. 4. Instituto de Meio Ambiente de Mato Grosso do Sul/IMASUL, Rua Des. Leão do Carmo s/n², Parque dos Poderes, 79.031-902, Campo Grande, MS, Brasil.
\end{abstract}

Recebido 12 dezembro 2016

Aceito 6 fevereiro 2017

DOI: 10.1590/1678-4766e2017107

\begin{abstract}
Oligochaeta (Annelida) of continental aquatic environments from Mato Grosso do Sul (Brazil). This study aimed a survey of Oligochaeta species in three different regions of Mato Grosso do Sul state (Baía, Ivinhema and Negro) in diverse environments (rivers, lakes, connecting channels). We recorded 41 Oligochaeta species, distributed in five families (Narapidae, Haplotaxidae, Opistocystidae, Alluroididae, and Naididae). The study showed Oligochaeta richness in river Negro regions in relation of regions degraded by human action.
\end{abstract}

KEYWORDS. Richness, zoobentic, floodplain, Biota-MS Program.

RESUMO. Este trabalho teve como objetivo apresentar um levantamento das espécies de Oligochaeta em três regiões diferentes do estado do Mato Grosso do Sul (Baía, Ivinhema e Negro) em diversos ambientes (rios, lagoas, canais de ligação). Foram registradas 41 espécies de Oligochaeta, distribuídas em 5 famílias (Narapidae, Haplotaxidae, Opistocystidae, Alluroididae e Naididae). O estudo mostrou a riqueza de Oligochaeta na região do rio Negro em relação às regiões mais degradadas pela ação humana.

PALAVRAS-CHAVE. Riqueza, zoobentos, planície de inundação, Programa Biota-MS.

Oligochaeta é um grupo cujos pequenos espécimes habitam quase todos os nichos dos ecossistemas de água doce (Marchese, 1995; TAKeda \& Fujita, 2004), comumente encontrado em diversos corpos de água como as de bromélias, entre folhas e troncos de árvores em decomposição e sedimentos (BrinkHURST \& JAMIESON, 1971; GAVRILOV, 1977; MARCHESE, 1995).

Os primeiros registros de Oligochaeta em ambientes aquáticos brasileiros datam do início do século XX, mas somente entre as décadas de 1940 e 1960 é que houve um maior conhecimento sobre os oligoquetos límnicos no Brasil, devido aos trabalhos realizados pelos pesquisadores Marcus e Du Bois-Reymond Marcus (Righi, 2002).

Entre as décadas de 1970 e 1990, os estudos realizados pelo professor Gilberto Righi consolidaram o atual conhecimento da taxonomia e sistemática de Oligochaeta no Brasil (MoReno \& Mischis, 2004; RömbKe, 2004). Desde então, alguns trabalhos sobre a composição, distribuição e ecologia dos Oligochaeta aquáticos têm sido realizados (Takeda, 1999; Alves \& Strixino, 2000; MontanholiMartins \& TaKeda, 2001; Collado \& Schmelz, 2001; Corbi \& Trivinho-Strixino, 2002, entre outros).

Na planície de inundação do alto rio Paraná,
Oligochaeta é também um dos grupos mais abundantes, ocorrendo em diferentes ambientes (TAKEDA, 1999; TAKEDA \& FUJITA, 2004). Estudos desse grupo nessa planície vêm sendo realizados desde 1986, indicando que o ciclo hidrológico, a proximidade com o canal principal, tipo de sedimento e quantidade de matéria orgânica são importantes fatores que exercem influência sobre a variação espacial e temporal da abundância desses organismos (TAKEDA, 1999; MONTANHOLIMARTINS \& TAKEDA, 1999, 2001).

Muitos estudos sobre a ocorrência, distribuição e riqueza de macroinvertebrados bentônicos foram feitos com insetos aquáticos e de crustáceos decápodos, mas raramente incluem o grupo de Oligochaeta (Alves et al., 2008). Objetiva-se apresentar um levantamento das espécies de Oligochaeta de diversos ambientes aquáticos do estado do Mato Grosso do Sul.

\section{MATERIAL \& MÉTODOS}

Área de estudo. A região do rio Negro faz divisa entre Corguinho e Rio Negro e entre o município de Rio Negro e Rio Verde de Mato Grosso e atravessa o Pantanal de Aquidauana. A bacia do alto-rio-Negro é formada por 
nascentes localizadas nos municípios de Rio Negro. O rio flui para a planície pantaneira da qual é um dos principais provedores. Plantas aquáticas são comuns e abundantes em lagoas da região do médio e baixo rio Negro. Bancos de macrófitas não são frequentes nos canais principais e em quase toda área e rios da pesquisa (FosTER et al., 2000).

A região do rio Baía, de fluxo lento, é um ambiente conectado ao rio Paraná através de um canal na sua porção inferior. Ele apresenta canais sinuosos de variadas larguras, uma profundidade média de 3,2 m, uma baixa declividade e baixa corrente e está influenciado pelo regime hidrológico do rio Paraná (Thomaz et al., 1991).

A região do rio Ivinhema é localizada nos municípios de Naviraí, Taquarussu e Jateí e é onde o primeiro parque foi criado no estado de Mato Grosso do Sul. Os varjões do Parque do Ivinhema compreendem um dos últimos trechos livres de represamentos de tributários do rio Paraná. É uma área de inundações periódicas, que protege refúgios de espécies de animais e vegetais do cerrado, e da floresta estacional (CESP, 2010). O rio Ivinhema no trecho amostrado tem profundidade média em torno de $3,9 \mathrm{~m}$, velocidade de corrente de 0,85 $\mathrm{m} . \mathrm{s}^{-1}$ e é um dos principais afluentes da margem direita do rio Paraná (Souza Filho \& Stevaux, 1997). (veja Tab. I para uma lista dos ambientes amostrados e suas respectivas coordenadas geográficas).

Amostragem. Na planície de inundação do alto rio Paraná, as amostras foram coletadas trimestralmente em 2000, 2001 e 2010 em 24 estações de coleta (lagoas de inundação, canais secundários e canal principal) e de 2002 a 2007 em oito estações de coleta (selecionados a partir de 24 estações de coleta), nas proximidades do rio Ivinhema e rio Baía. $\mathrm{Na}$ região do rio Negro, foram realizadas amostragens em nove estações de coleta, em 1998.

Para as amostragens bênticas foi utilizado um pegador de fundo tipo Petersen modificado e posteriormente realizada a lavagem do material coletado em uma série de peneiras de malhas: $2,0 \mathrm{~mm} ; 1,0 \mathrm{~mm}$ e $0,2 \mathrm{~mm}$, sendo os organismos retidos nas duas primeiras malhas retirados e imediatamente fixados em álcool $80 \%$. O sedimento retido na última peneira também foi fixado com álcool $80 \%$ para a posterior triagem sob microscópio estereoscópico. Após a triagem, as espécies de Oligochaeta foram identificadas utilizando-se as chaves de BRINKHURST \& JAMIESON (1971), Righi (1984) e BrinKHURST \& MARCHESE (1991). O material está depositado no Laboratório de Zoobentos do Núcleo de Pesquisas em Limnologia, Ictiologia e Aquicultura (Nupelia) da Universidade Estadual de Maringá (Maringá, Paraná).

\section{RESULTADOS E DISCUSSÃO}

Foram registradas 41 espécies de Oligochaeta, distribuídas em cinco famílias (Narapidae, Haplotaxidae, Opistocystidae, Alluroididae e Naididae). Naididae foi a mais representativa, com 36 espécies pertencentes a Naidinae (19 espécies), Pristininae (10 espécies) e Tubificinae (7 espécies). Em relação a Narapidae, Haplotaxidae e Alluroididae, foi registrada apenas uma espécie para cada, e para Opistocystidae, duas espécies. De acordo com RigHI (2002), são atualmente conhecidas no Brasil cerca de 70 espécies de Oligochaeta, portanto o registro de 41 espécies em apenas um estado é um número considerável.

Naididae foi a família mais representativa nas três regiões amostradas, enquanto Haplotaxidae registrada apenas na região do rio Ivinhema. Na região do rio Baía, além de Naididae foram encontradas apenas Opistocystidae e Alluroididae. Na região do rio Ivinhema todas as famílias que ocorreram nesse estudo foram registradas, enquanto na região do rio Negro apenas Haplotaxidae não foi encontrada (Fig. 1).

Tab. I. Coordenadas geográficas dos ambientes amostrados do Estado do Mato Grosso do Sul, Brasil

\begin{tabular}{|c|c|c|c|c|c|}
\hline \multicolumn{2}{|c|}{ Região do rio Baía } & \multicolumn{2}{|c|}{ Região do rio Ivinhema } & \multicolumn{2}{|c|}{ Região do rio Negro } \\
\hline Lagoa Aurélio & $\begin{array}{l}22^{\circ} 41^{\prime} 40.3^{\prime \prime} \mathrm{S} \\
53^{\circ} 13^{\prime} 55.8^{\prime \prime} \mathrm{W}\end{array}$ & Lagoa Boca do Ipoitã & $\begin{array}{l}22^{\circ} 50^{\prime} 05.2^{\prime \prime} \mathrm{S}, \\
53^{\circ} 33^{\prime} 54.44^{\prime} \mathrm{W}\end{array}$ & Corixo Santo Antônio & $\begin{array}{l}19^{\circ} 36.16^{\prime \prime} \mathrm{S}, \\
56^{\circ} 19.72^{\prime \prime} \mathrm{W}\end{array}$ \\
\hline Canal Baía & $\begin{array}{l}22^{\circ} 41^{\prime} 27.3 " \mathrm{~S}, \\
53^{\circ} 13^{\prime} 30.0^{\prime \prime} \mathrm{W}\end{array}$ & Lagoa Capivara & $\begin{array}{c}22^{\circ} 47^{\prime} 58.9 " \mathrm{~S}, \\
53^{\circ} 32^{\prime} 04.60 " \mathrm{~W}\end{array}$ & Brejo Santa Sofia & $\begin{array}{l}19^{\circ} 35.89^{\prime \prime} \mathrm{S}, \\
56^{\circ} 20.47^{\prime \prime} \mathrm{W}\end{array}$ \\
\hline Canal Curutuba & $\begin{array}{l}22^{\circ} 45^{\prime} 05.8^{\prime \prime} \mathrm{S}, \\
53^{\circ} 21^{\prime} 35.1^{\prime \prime} \mathrm{W}\end{array}$ & Lagoa Cervo & $\begin{array}{c}22^{\circ} 46^{\prime} 33.5^{\prime \prime} \mathrm{S}, \\
53^{\circ} 29^{\prime} 51.80^{\prime \prime} \mathrm{W}\end{array}$ & Rio Novo & $\begin{array}{l}19^{\circ} 36.64^{\prime \prime} \mathrm{S}, \\
56^{\circ} 24.87^{\prime \prime} \mathrm{W}\end{array}$ \\
\hline Lagoa Fechada & $\begin{array}{l}22^{\circ} 42^{\prime} 40.7^{\prime \prime} \mathrm{S}, \\
53^{\circ} 16^{\prime} 38.4^{\prime \prime} \mathrm{W}\end{array}$ & Lagoa Finado Raimundo & $\begin{array}{l}22^{\circ} 47^{\prime} 44.4 " \mathrm{~S}, \\
53^{\circ} 32^{\prime} 17.60^{\prime \prime} \mathrm{W}\end{array}$ & Baía Bonita & $\begin{array}{l}19^{\circ} 34.04^{\prime \prime} \mathrm{S}, \\
56^{\circ} 14.06^{\prime \prime} \mathrm{W}\end{array}$ \\
\hline Lagoa Gavião & $\begin{array}{l}22^{\circ} 40^{\prime} 45.6^{\prime \prime} \mathrm{S}, \\
53^{\circ} 13^{\prime} 51.0^{\prime \prime} \mathrm{W}\end{array}$ & Canal Ipoitã & $\begin{array}{l}22^{\circ} 50^{\prime} 15.4 " \mathrm{~S}, \\
53^{\circ} 33^{\prime} 32.52^{\prime} \mathrm{W}\end{array}$ & Lagoa Salina & $\begin{array}{l}19^{\circ} 33.30^{\prime \prime} \mathrm{S}, \\
56^{\circ} 13.44^{\prime \prime} \mathrm{W}\end{array}$ \\
\hline Lagoa Guaraná & $\begin{array}{l}22^{\circ} 43^{\prime} 10.3 " \mathrm{~S}, \\
53^{\circ} 18^{\prime} 02.6^{\prime \prime} \mathrm{W}\end{array}$ & Rio Ivinhema & $\begin{array}{l}22^{\circ} 48^{\prime} 14.3^{\prime \prime} \mathrm{S}, \\
53^{\circ} 32^{\prime} 46.75^{\prime} \mathrm{W}\end{array}$ & Rio Negro & $\begin{array}{l}19^{\circ} 36.70^{\prime \prime} \mathrm{S}, \\
56^{\circ} 02.78^{\prime \prime} \mathrm{W}\end{array}$ \\
\hline Lagoa Maria Luíza & $\begin{array}{l}22^{\circ} 40^{\prime} 27.6 " \mathrm{~S}, \\
53^{\circ} 13^{\prime} 10.1^{\prime \prime} \mathrm{W}\end{array}$ & Lagoa Jacaré & $\begin{array}{l}22^{\circ} 47^{\prime} 02.1 " \mathrm{~S}, \\
53^{\circ} 29^{\prime} 58.60 " \mathrm{~W}\end{array}$ & Rio Vermelho & $\begin{array}{l}19^{\circ} 37.22^{\prime \prime S}, \\
56^{\circ} 57.27^{\prime \prime} \mathrm{W}\end{array}$ \\
\hline Lagoa Onça & $\begin{array}{l}22^{\circ} 39^{\prime} 51.4^{\prime \prime} \mathrm{S}, \\
53^{\circ} 12^{\prime} 02.1^{\prime \prime} \mathrm{W}\end{array}$ & Lagoa dos Patos & $\begin{array}{l}22^{\circ} 49^{\prime} 21.2{ }^{\prime} \mathrm{S}, \\
53^{\circ} 33^{\prime} 10.03 ” \mathrm{~W}\end{array}$ & Rio Abobral & $\begin{array}{l}19^{\circ} 26.68^{\prime \prime} \mathrm{S}, \\
57^{\circ} 02.49^{\prime \prime} \mathrm{W}\end{array}$ \\
\hline Lagoa Pousada das Garças & $\begin{array}{l}22^{\circ} 42^{\prime} 00.8^{\prime \prime} \mathrm{S}, \\
53^{\circ} 15^{\prime} 24.8^{\prime \prime} \mathrm{W}\end{array}$ & Lagoa Peroba & $\begin{array}{l}22^{\circ} 54^{\prime} 29.5^{\prime \prime} \mathrm{S}, \\
53^{\circ} 38^{\prime} 23.27^{\prime} \mathrm{W}\end{array}$ & Rio Negrinho & $\begin{array}{l}19^{\circ} 20.29^{\prime \prime} \mathrm{S}, \\
56^{\circ} 57.70^{\prime \prime} \mathrm{W}\end{array}$ \\
\hline Lagoa dos Porcos & $\begin{array}{l}22^{\circ} 42^{\prime} 05.3^{\prime \prime S}, \\
53^{\circ} 14^{\prime} 40.7^{\prime \prime} \mathrm{W}\end{array}$ & Lagoa Sumida & $\begin{array}{l}22^{\circ} 47^{\prime} 02.6 " \mathrm{~S}, \\
53^{\circ} 29^{\prime} 30.87^{\prime \prime} \mathrm{W}\end{array}$ & & \\
\hline Rio Baía & $\begin{array}{l}22^{\circ} 43^{\prime} 23.1^{\prime \prime} \mathrm{S}, \\
53^{\circ} 17^{\prime} 37.1 ' \mathrm{~W}\end{array}$ & Lagoa Ventura & $\begin{array}{c}22^{\circ} 51^{\prime} 34.5 " \mathrm{~S}, \\
53^{\circ} 36^{\prime} 04.54 " \mathrm{~W}\end{array}$ & & \\
\hline Lagoa Traíra & $\begin{array}{l}22^{\circ} 44^{\prime} 47.8^{\prime \prime} \mathrm{S}, \\
53^{\circ} 20^{\prime} 22.5^{\prime \prime} \mathrm{W}\end{array}$ & Lagoa Zé do Paco & $\begin{array}{c}22^{\circ} 50^{\prime} 04.2^{\prime \prime} \mathrm{S}, \\
53^{\circ} 34^{\prime} 19.85^{\prime \prime} \mathrm{W}\end{array}$ & & \\
\hline
\end{tabular}


Pristina americana, Aulodrulis pigueti, Slavina evelinae e Pristina proboscidae foram os táxons mais frequentes, ocorrendo na maior parte dos ambientes amostrados o que pode demonstrar a alta plasticidade ambiental dessas espécies. Entretanto, espécies como Dero (Aulophorus) costatus, Dero (Aulophorus) lodeni, Dero (Aulophorus) hymanae, Dero (Aulophorus) vaga, Nais variabilis e Aulodrilus adetus foram espécies menos frequentes, com ocorrência restrita a apenas uma região (Fig. 2).

Dentre os ambientes amostrados, alguns se destacam por apresentar maior número de espécies em relação aos demais. Na região do rio Baía, os ambientes de maior riqueza foram rio Baía (19 espécies) e o canal Curutuba (22 espécies), os quais têm médio fluxo de água; na região do rio Ivinhema, o rio Ivinhema (22 espécies) e lagoa Ventura (19 espécies); e na região rio Negro, o rio Abobral, com 17 espécies registradas (Tab. II).

Nos rios Ivinhema, Abobral, Vermelho e canal Ipoitã, Narapa bonettoi e Haplotaxis aedochaeta foram registradas. Essas espécies estão adaptadas a viver entre os interstícios dos grãos de areia com altas velocidades de fluxo, condições típicas de ambientes lóticos (MontanHOLI-Martins \& TAKEDA, 2001; BLETTER et al., 2008).

Foram registrados elevados valores de riqueza de Oligochaeta, com a região do rio Baía com 28 espécies, região do rio Ivinhema com 31 e região do rio Negro com 25 espécies. Algumas espécies foram registradas apenas
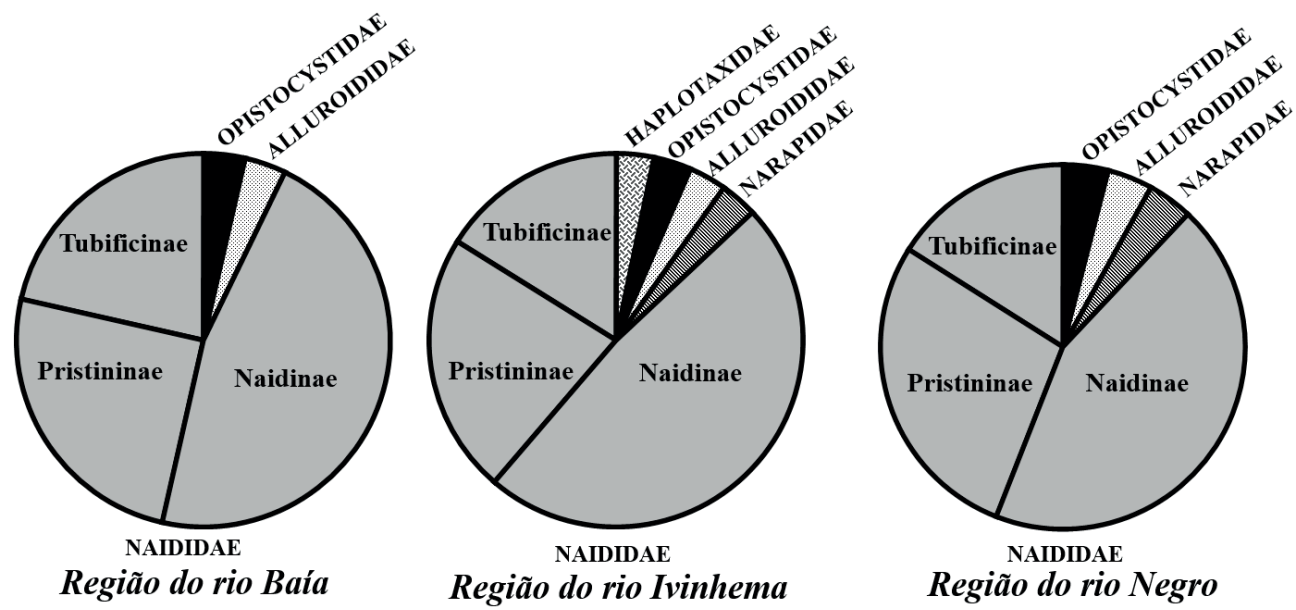

Fig. 1. Número de espécies encontradas em cada família e subfamília nas três regiões amostradas no Estado do Mato Grosso do Sul, Brasil.

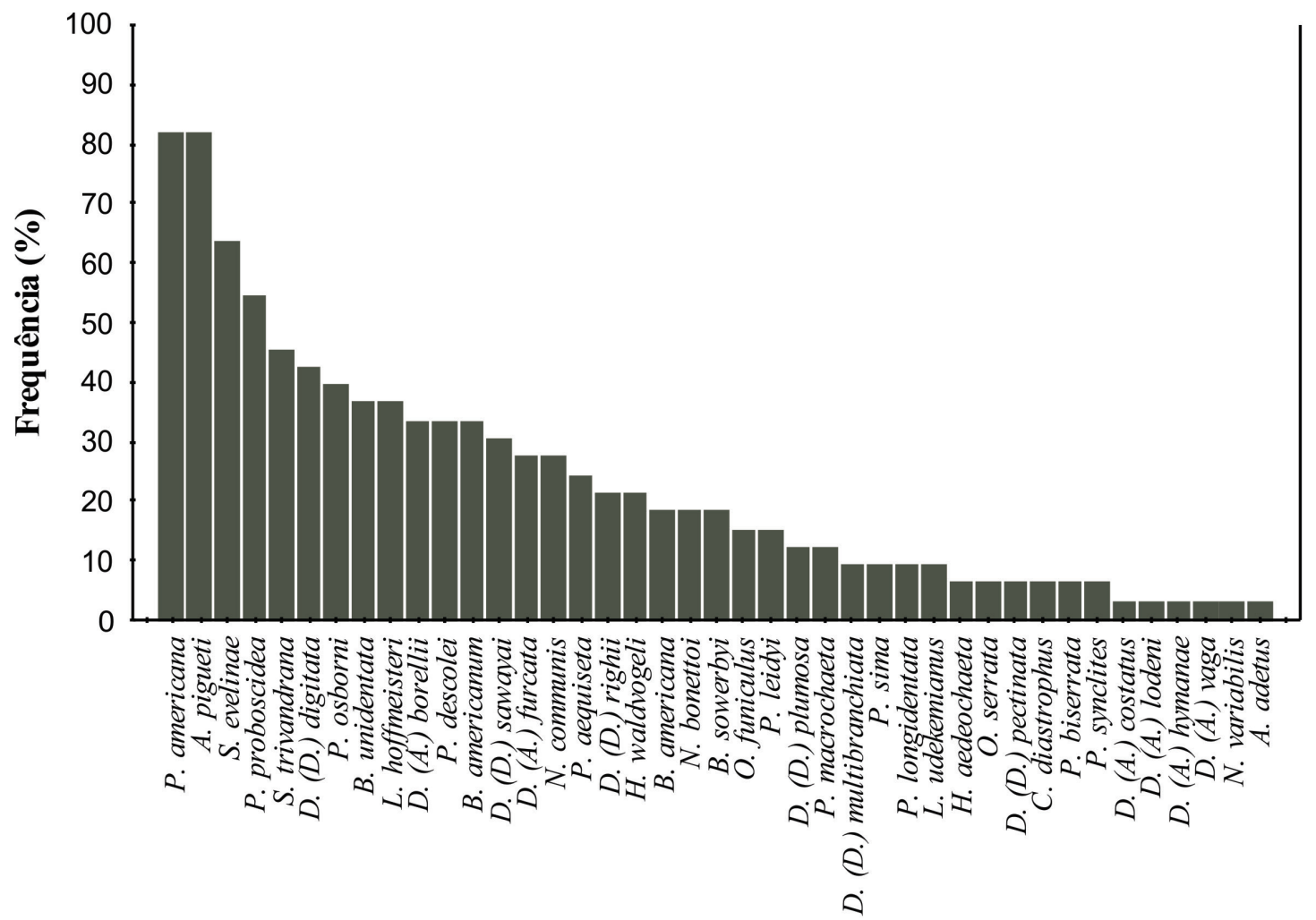

Fig. 2. Frequência de ocorrência (\%) das espécies de Oligochaeta registradas nos 33 ambientes amostrados do estado do Mato Grosso do Sul, Brasil. 
Tab. II. Lista de espécies de Oligochaeta nos 33 ambientes amostrados do estado do Mato Grosso do Sul, Brasil. (1, Lagoa Aurélio; 2, Canal Baía; 3, Canal Curutuba; 4, Lagoa Fechada; 5, Lagoa Gavião; 6, Lagoa Guaraná; 7, Lagoa Maria Luíza; 8, Lagoa Onça; 9, lagoa Pousada das Garças; 10, Lagoa dos Porcos; 11, Rio Baía; 12, Lagoa Traíra; 13, Lagoa Boca do Ipoitã; 14, Lagoa Capivara; 15, Lagoa Cervo; 16, Lagoa Finado Raimundo; 17, Canal Ipoitã; 18, Rio Ivinhema; 19, Lagoa Jacaré; 20, Lagoa dos patos; 21, Lagoa Peroba; 22, Lagoa Sumida; 23, Lagoa Ventura; 24, Lagoa Zé do Paco; 25, Corixo Santo Antônio; 26, Brejo Santa Sofia; 27, Rio Novo; 28, Baía Bonita; 29, Lagoa Salina; 30, Rio Negro; 31, Rio Vermelho; 32, Rio Abobral; 33, Rio Negrinho).

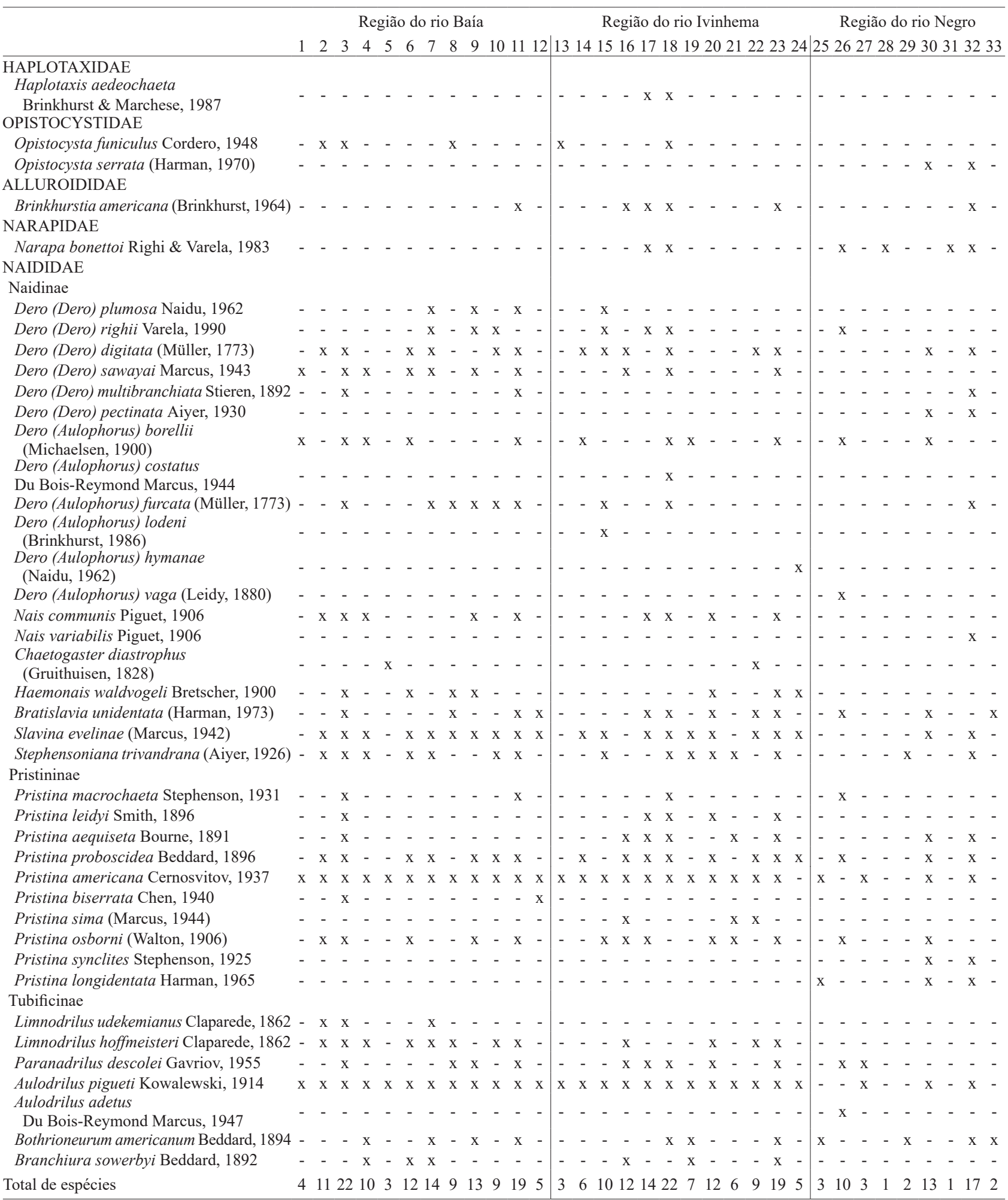


em uma das regiões, como P. bisserata e L. udenkemianus na região rio Baía; $H$. aedochaeta, D. (A.) costatus, D. (A.) lodeni, $D$. (A.) hymanae, $P$. sima na região do rio Ivinhema e O. serrata, D. (D.) pectinata, D. (A.) vaga, N. variabilis, P. synclites, . longidenata, na região do rio Negro (Fig. 3).

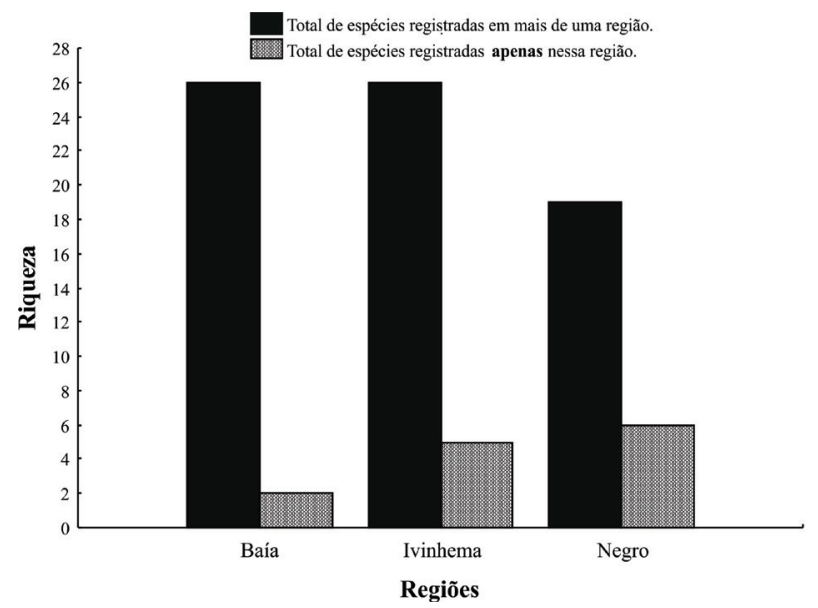

Fig. 3. Riqueza de Oligochaeta encontradas em apenas uma determinada região ou em mais de uma região, estado do Mato Grosso do Sul, Brasil.

Esses resultados demonstram a importância desses corpos de água para a manutenção da riqueza de Oligochaeta no estado do Mato Grosso do Sul. Na região do rio Negro, apesar de ter sido realizada apenas uma coleta, foi possível observar elevados valores de riqueza e de ocorrência exclusiva dessa região, aproximando o registro das regiões do rio Baía e rio Ivinhema coletados em 11 anos de monitoramento contínuo. Muitas espécies de Tubificinae vêm desaparecendo pouco a pouco ao longo dos anos na planície de inundação do alto rio Paraná, como Bothrioneurum americanum, Paranadrilus descolei e Branchiura sowerbyi antes abundantes e hoje muito raramente encontradas. Para esta redução, pode-se sugerir - apesar da distância - a influência da construção da barragem de Porto Primavera, e que vem afetando toda comunidade bentônica, inclusive a assembleia de Oligochaeta, indicando que essas espécies são excelentes sentinelas de mudanças ambientais.

Agradecimentos. Agradecemos a Fundação de Apoio ao Desenvolvimento do Ensino, Ciências e Tecnologia do Estado de Mato Grosso do Sul (Fundect) e a Superintendência de Ciências e Tecnologia do Estado de Mato Grosso do Sul (Sucitec/MS), pelo convite de participação neste fascículo especial da Iheringia, Série Zoologia e o suporte financeiro para sua publicação. E o financiamento e apoio do Pantanal AquaRAP/ Conservation International-Pantanal em 1998 e dos projetos CNPq/PELD/ UEM/ Nupelia (Proc. 0230/98 e 4280/2010).

\section{REFERÊNCIAS BIBLIOGRÁFICAS}

Alves, R. G. \& Strixino, G. 2000. Distribuição espacial em uma lagoa marginal do Rio Mogi-Guaçu. Iheringia, Série Zoologia (88):173-180.
Alves, R. G.; Marchese, M. R. \& Martins, R. T. 2008. Oligochaeta (Annelida, Clitellata) of lotic environments at Parque Estadual Intervales (São Paulo, Brazil). Biota Neotropica 8(1):69-72.

Bletter, M.; Amsler, M.; Ezcurra de Drago, I. \& Marchese, M. 2008. Effects of stream hydraulics and other environmental variables on density of Narapa bonettoi (Oligochaeta) in the Paraná River system. River Research and Applications 14(8):1124-1140.

Brinkhurst, R. O. \& Jamieson, B. M. G. 1971. Aquatic Oligochaeta of the world. Edinburgh, Oliver and Boyd. 860p.

BRINKHURST, R. O. \& MARCHESE, M. R. 1991. Guia para la identificación de oligoquetos aquáticos continentales de Sud y Centro América. Santo Tomé, Asociación de Ciencias Naturales del Litoral. 549p.

CESP (Companhia Elétrica do Estado de São Paulo). Disponível em: $<$ http://www.cesp.com.br/portalCesp/biblio.nsf/V03.01/unidades conservacao/\$file/unidade_conservacao.pdf $>$. Acesso em 14.09.2012.

Collado, R. \& Schmelz, R. M. 2001. Descriptions of three Pristina species (Naididae, Clitellata) from Amazonian forest soils, including P. marcusi sp. nov. Hydrobiologia 463:1-11.

Corbi, J. J. \& Trivinho-Strixino, S. 2002. Spatial and bathymetric distribution of the macrobenthic fauna of the Ribeirão das Anhumas Reservoir (Américo Brasiliense-SP, Brazil). Acta Limnogica Brasiliensia 14(1):35-42.

Foster, R. B.; PotT, V. J. \& Salis, S. D. 2000. Description of the Vegetation of the Rio Negro, Pantanal, Mato Grosso do Sul, Brasil. In: WillinK, P. W.; Chernoff, B.; Alonso, L. E.; Montanbault, J. R. \& Lourival, R. eds. A biological Assessment of the Aquatic Ecosystems of the Pantanal, Mato Grosso do Sul, Brasil. Conservation International. 306 .

Gavrilov, K. 1977. Oligochaeta. In: Hurbertu, S. H. ed. Biota Acuática de Sudamérica Austral. San Diego, San Diego State University, p.99-121.

Marchese, M. 1995. Annelida Oligochaeta. In: Lopreto, E. \& Tell, G. eds. Ecosistemas de aguas continentales. Metodologías para su estudio. La Plata, Ediciones Sur. Tomo II, p.709-731.

Montanholi-Martins, M. C. \& TaKeda, A. M. 1999. Communities of benthic oligochaetes in relation to sediment structure in the Upper Paraná River, Brazil. Studies on Neotropical Fauna Environment 34:52-58.

Montanholi-Martins, M. C. \& TAKeda, A. M. 2001. Spatial and temporal variations of oligochaetes of the Ivinhema River and Patos Lake in the Upper Paraná River Basin, Brazil. Hydrobiologia 463:197-205.

Moreno, A. G. \& Mischis, C. C. 2004. The status of Gilberto Righi's collection at the museum of São Paulo. Righi memorial: tropical ecology. Pedobiologia 47(5-6):413-418.

RighI, G. Oligochaeta. 1984. In: SCHADEN, R. ed. Manual de identificação de invertebrados límnicos do Brasil. Brasília, CNPq. 48p.

Righi, G. 2002. Anelídeos Oligoquetos. In: Ismael, D.; Valenti, W. C.; Matsumura-Tundisi, T. \& Rocha, O. eds. Biodiversidade do Estado de São Paulo, Brasil. Invertebrados de água doce. São Paulo, FAPESP. vol. 4, p. 81-84.

RömBKE, J. 2004. The role of Gilberto Righi in the development of tropical taxonomy. Pedobiologia 47(5-6):405-412.

Souza Filho, E. E. \& Stevaux, J. C. 1997. Geologia e geomorfologia do complexo rio Baia, Curutuba e Ivinheima. In: Vazzoler, A. E. A. M.; Agostinho, A. A. \& Hahn, N. S. eds. Planície de Inundação do Alto rio Paraná. Maringá, EDUEM. 460p.

TAKEDA, A. M. 1999. Oligochaeta community of alluvial upper Paraná River, Brazil: Spatial and temporal distribution (1987-1988). Hydrobiologia 412:35-42.

TAKedA, A. M. \& Fujita, D. S. 2004. Benthic invertebrates. In: Thomaz, S. M.; Agostinho, A. A. \& Hahn, N. S. eds. The Upper Paraná River and its Floodplain. Leiden, Backhuys Publishers, p. 192-207.

Thomaz, S. M.; Roberto, M. C.; LansaC-Toha, F. A.; Esteves, F. A. \& LimA, A. F. 1991. Dinâmica temporal dos principais fatores limnológicos do rio Baía-planície de inundação do alto rio Paraná-MS, Brasil. Revista Unimar 13(2):299-312. 